\section{A case of diffuse infiltrative lung disease with chronic vomiting after gastrectomy}

\section{Case history}

A 47-year-old male was referred to the Respiratory Division of the Kyoto-Katsura Hospital (Kyoto, Japan) with chronic coughing, vomiting and a slight fever. The patient had a history of a gastrectomy and reported that he would vomit several times after going to bed at night when he had overeaten. The frequency of vomiting had increased in recent months, and he had started coughing when vomiting. Approximately 1 week before he presented himself, he was seen by a primary care physician due to fever and was empirically administered antibiotics. At this time, he was vomiting almost daily. He was an ex-smoker with a 75 pack-year smoking history and had quit smoking 2 years previously. His physical examination was within normal limits, as were pulmonary function tests. Laboratory investigations revealed blood leukocytosis of $14.1 \times 10^{9}$ cells per $L(73 \%$ neutrophils) and C-reactive protein levels were elevated at $58 \mathrm{mg}$ per L. Repeated smear examinations of sputum samples for acid-fast bacilli (AFB) were negative despite neutrophilia, and only normal flora grew in culture. A rapid species identification test was performed with sputum through commercial DNA probes using PCR, but sputum was negative for Mycobacterium tuberculosis and Mycobacterium avium-intracellurare.

We consulted the surgeon that had operated on this subject, and discovered he had undergone a proximal gastrectomy, a jejunum pouch reconstruction, a cholecystectomy, and a splenectomy to surgically treat T2N1MO (tumor 2, node 1 , metastasis 0 ) gastric cancer. A chest radiograph performed 3 months prior to the present investigation was compared with one taken on the subject's first visit to an outpatient clinic.

\section{Task 1}

Interpret the chest radiographs

(figure 1).
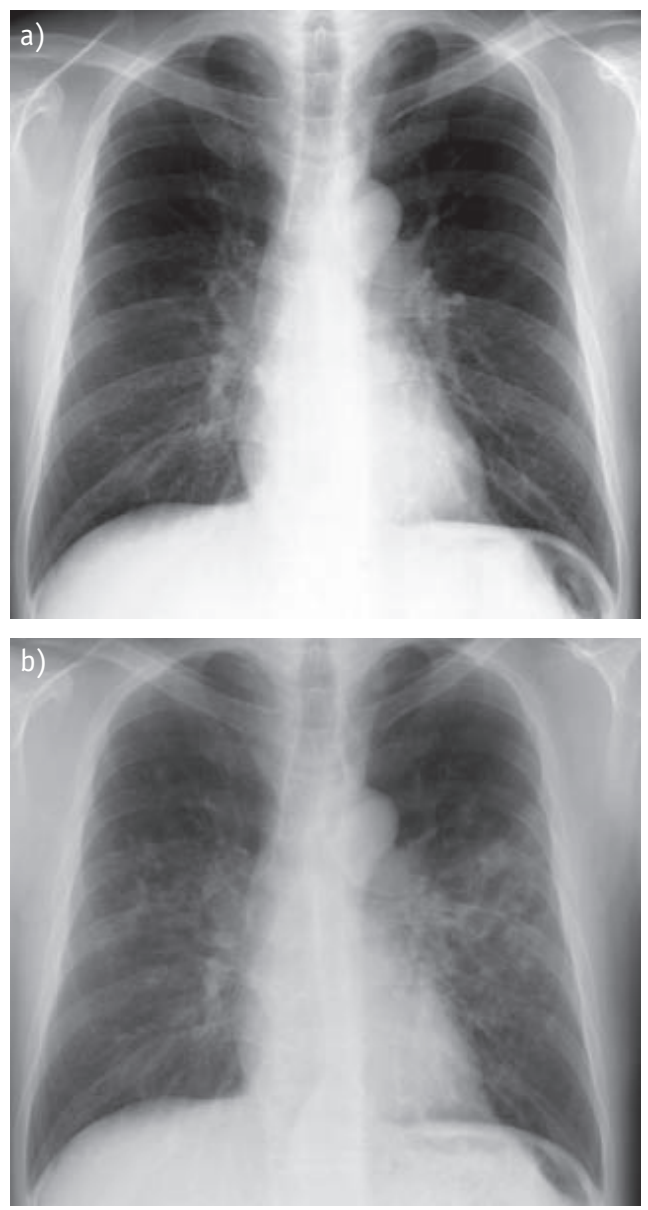

Figure 1

Chest radiographs taken a) post-surgery, 3 months prior to the current admission and b) on the subject's first visit to an outpatient clinic.

\section{K. Nishimura ${ }^{1}$ \\ M. Okamoto ${ }^{2}$ \\ M. Santo ${ }^{3}$ \\ T. Hanawa ${ }^{3}$ \\ T. Nishimura ${ }^{3}$ \\ T. Oga ${ }^{4}$}

${ }^{1}$ Dept of Respiratory Medicine, Murakami Memorial Hospital, Asahi University, Gifu, ${ }^{2}$ First Dept of Internal Medicine, Kansai Medical University, Hirakata, ${ }^{3}$ Kyoto-Katsura Hospital, Kyoto, and ${ }^{4}$ Dept of Respiratory Care and Sleep Regulation, Graduate School of Medicine, Kyoto University, Kyoto, Japan

\section{Correspondence}

K. Nishimura

Dept of Respiratory Medicine Murakami Memorial Hospital Asahi University

3-23 Hashimoto-cho

Gifu, 500-8523

Japan

koichi-nishimura@nifty.com

\section{Provenance}

Submitted article, peer reviewed

\section{Competing interests}

K. Nishimura has received lecture fees and research grants from Boehringer-Ingelheim, GlaxoSmithKline and Teijin Pharma, and is an advisor for Boehringer-Ingelheim, but not in relation to the topic of the current manuscript.

HERMES syllabus link: modules B.4.5, D.2.1, D.2, D.3.1 


\section{Answer 1}

The first radiograph shows no abnormalities. The second, however, shows bilateral heterogeneous areas of increased opacity in the middle and lower lungs.

High-resolution computed tomography (HRCT) was performed (figure 2).

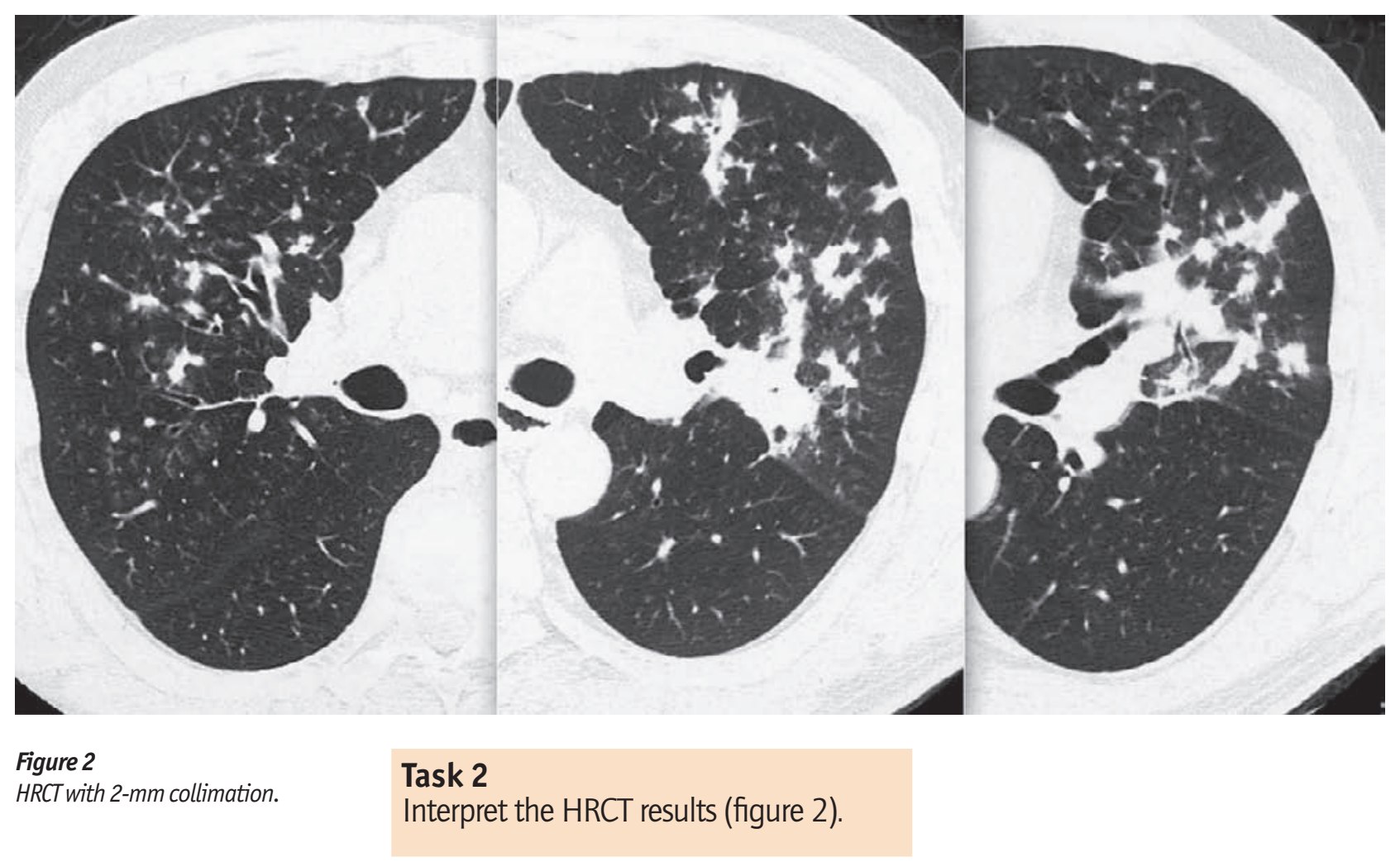




\section{Answer 2}

The HRCT image shows small nodular areas of increased opacity along vessels (partially tree-in-bud appearance), thickened branching linear opacities and multifocal brochiectasis.

The test findings resulted in the hospitalisation of the patient for 12 days to undergo a bronchoscopy for a presumptive diagnosis of aspiration pneumonia. The patient was not allowed to orally ingest solid foods or liquids, and was intravenously given $600 \mathrm{mg}$ of clindamycin hydrochloride (CLDM) twice a day. Symptoms and inflammatory responses seemed to improve slightly, but the result was unclear, and chest radiography findings remained unchanged. The patient underwent a bronchoscopy during his hospitalisation. Bronchoalveolar lavage (BAL) was performed in the lingula, and $70 \%$ of 150 $\mathrm{mL}$ saline was recovered with cell differentiation of $75 \%$ macrophages and $25 \%$ lymphocytes (the $\mathrm{CD} 4+/ \mathrm{CD} 8+$ ratio was 2.56 ). Gross and histological examinations of samples obtained from BAL fluid sediments and transbronchial lung biopsies (TBLB) did not reveal foreign bodies or food residue. Histological examinations of lung specimens collected in TBLB were all nonspecific.

The patient was discharged without any evidence to support the diagnosis of aspiration pneumonia. The patient continued to vomit and had a fever after his discharge, while his inflammatory response increased. He was admitted to the hospital 1 month later for a surgical lung biopsy.

\section{Task 3}

What would be your diagnosis?

a) Lymphangitis carcinomatosa

b) Nontuberculous mycobacterial lung disease

c) Aspiration pneumonia, despite the lack of evidence

d) Cystic fibrosis 


\section{Answer 3}

a) This is an incorrect answer.

b) This is the correct answer. The patient has nontuberculous mycobacterial (NTM) lung disease due to rapidly growing mycobacteria (RGM): a lung infected with Mycobacterium fortuitum.

c) This is an incorrect answer.

d) This is an incorrect answer.

\section{Clinical course}

Immediately after the patient was admitted for the second time to receive a surgical lung biopsy, we were informed that there was a slight growth of AFB in sputum samples that had been submitted in previous examinations. We decided not to conduct a surgical biopsy and waited until complete culture results became available. Two weeks after the patient first saw us, the following combination of antibiotics was started: rifampin, ethambutol, isoniazid and clarithromycin. Bacteriological studies and 16S ribosomal RNA sequence analyses identified the cultured AFB as $M$. fortuitum. Before the administration of a combination of antibiotics, seven expectorated sputum samples, three stomach fluid samples, and one bronchial lavage sample were submitted to test for AFB, but all smear samples were negative. Cultures from four expectorated sputum samples and one bronchial lavage sample were positive. Once the diagnosis of a $M$. fortuitum lung infection became definite, the combination of antibiotics was changed to ofloxacin and clarithromycin. After more than 9 months of treatment and confirmation of negative sputum cultures, the patient returned to the care of a general practitioner.

\section{Discussion}

This was a special case in which the patient vomited daily, and the initial diagnosis of aspiration pneumonia may have been likely, even if the association between NTM pulmonary disease due to RGM and chronic vomiting had been previously documented in the respiratory literature. However, the specific presentation of this rare disease is not widely known, and we would have been able to diagnose this patient more easily if we had a priori knowledge of this association. Chest physicians should consider the likelihood of rapidly growing AFB, including $M$. fortuitum, when presented with lung lesions associated with chronic vomiting.
Since GiBson [1] reported a case of achalasia associated with an NTM infection in 1953, similar findings have been published. However, it is rare to encounter lung lesions due to RGM such as $M$. fortuitum in clinical settings $[2,3]$. GRIF FाrH et al. [4] reported 154 cases in 1993: as far as we know, this is the largest number of cases reported. GRIFFTH et al. [4] reported that 10 cases had chronic vomiting associated with stomach and esophageal diseases, but details were not reported. An Official American Thoracic Society/ Infectious Diseases Society of America statement, published in 2007 [5], also discusses the association between chronic vomiting and lung lesions due to rapidly-growing AFB.

HADJILADIS et al. [6] reviewed 20 cases (including two cases of their own) of oesophageal or other swallowing disorders or lipoid pneumonia and complications due to RGM infections in 1999 [6]. Of these 20 cases, 11 had achalasia and four had other oesophageal diseases or dysphagia. The frequency of vomiting was not reported. M. fortuitum was isolated in 11 out of 15 cases in this report and in five out of 10 cases in the report of GRIFFTH et al. [4]. Four cases with gastrooesophageal problems in the report of HADIILADIS et al. [6] died, and VARGHESE et al. [7] also reported fatal cases. Almost all chest radiographic findings reported in these cases had bilateral infiltrative shadows. Church et al. [8] reported a similar case of a 51-year-old female who was vomiting due to oesophageal achalasia, but their first diagnosis and treatment were also based on the tentative diagnosis of aspiration pneumonia.

When we looked at literature from Asian countries, we found two reports respectively with 21 and 26 cases of $M$. fortuitum infections in Korea in 2006 and 2007 [9, 10], but the authors reported that none of the cases had gastric and/ or oesophageal conditions or chronic vomiting as complications. A Japanese literature search revealed approximately 30 cases of $M$. fortuitum infections [11, 12], but the only other similar case was reported by Hıкı et al. [13] in 2006 where a patient had oesophageal achalasia.

When a patient presents with diffuse infiltration associated with chronic vomiting, we must include $M$. fortuitum infections among the differential diagnoses. At the very least, expectorated sputum and other samples should always be tested for AFB. Based on these reports, this case was very characteristic, and a diagnosis may have been made more easily with a priori knowledge of the association. 


\section{References}

1. Gibson JB. Infection of the lungs by saprophytic mycobacteria in achalasia of the cardia, with report of a fatal case showing lipoid pneumonia due to milk. J Pathol Bacteriol 1953; 65: 239-251.

2. Dreisin RB, Scoggin C, Davidson PT. The pathogenicity of Mycobacterium fortuitum and Mycobacterium chelonei in man: a report of seven cases. Tubercle 1976; 57: 49-57.

3. Mornex JF, Guillot YL, Guerin JC, Kalb JC, Fougerat J. Lung disease due to Mycobacterium fortuitum. Lancet 1981; 1: 614.

4. Griffith DE, Girard WM, Wallace RJ Jr. Clinical features of pulmonary disease caused by rapidly growing mycobacteria. An analysis of 154 patients. Am Rev Respir Dis 1993; 147: 1271-1278.

5. Griffith DE, Aksamit T, Brown-Elliott BA, et al. An official ATS/IDSA statement: diagnosis, treatment, and prevention of nontuberculous mycobacterial diseases. Am J Respir Crit Care Med 2007; 175: 367-416.

6. Hadjiliadis D, Adlakha A, Prakash UB. Rapidly growing mycobacterial lung infection in association with esophageal disorders. Mayo Clin Proc 1999; 74: 45-51.

7. Varghese G, Shepherd R, Watt P, Bruce JH. Fatal infection with Mycobacterium fortuitum associated with oesophageal achalasia. Thorax 1988; 43: 151-152.

8. Church AC, Watkin S. Non-tuberculous mycobacteria masquerading as aspiration pneumonia in patients with gastrointestinal problems. Respir Med 2006; 100: 1663-1665.

9. Koh WJ, Kwon 0J, Jeon K, et al. Clinical significance of nontuberculous mycobacteria isolated from respiratory specimens in Korea. Chest 2006; 129: 341-348.

10. Park S, Suh GY, Chung MP, et al. Clinical significance of Mycobacterium fortuitum isolated from respiratory specimens. Respir Med 2008; 102: 437-442.

11. Tsukamura M, Kito K, Yamada T, Akiyama S. [Lung disease due to Mycobacterium fortuitum: report of 3 cases]. Kekkaku 1983; 585: 293-298.

12. Tsukamura M, Kita N, Shimoide H, Yoshimoto K, Wada T, Mitani Y. [Lung infections due to Mycobacterium fortuitum and Mycobacterium chelonae. Reports of nine cases of M. fortuitum infection and four cases of M. chelonae infection]. Kekkaku 1985; 60: 429-434.

13. Hiki, et al. [Report of proceedings.] Kekkaku 2006; 81: 500. 